\title{
Adhesive-free colloidal probes for nanoscale force measurements: Production and characterization
}

\author{
M. Indrieri, ${ }^{1}$ A. Podestà, ${ }^{1, a)}$ G. Bongiorno, ${ }^{2}$ D. Marchesi, ${ }^{2}$ and P. Milani ${ }^{1,2}$ \\ ${ }^{1}$ CIMaINa and Dipartimento di Fisica, Università degli Studi di Milano, via Celoria 16, 20133 Milano, Italy \\ ${ }^{2}$ Fondazione Filarete, Viale Ortles 22/4, 20139 Milano, Italy
}

(Received 8 December 2010; accepted 18 January 2011; published online 24 February 2011)

\begin{abstract}
We describe novel approaches for the production and characterization of epoxy- and adhesive-free colloidal probes for atomic force microscopy (AFM). Borosilicate glass microspheres are strongly attached to commercial AFM cantilevers exploiting the capillary adhesion force due to the formation of a water meniscus, and then a thermal annealing of the sphere-cantilever system at a temperature slightly below the softening point of borosilicate glass. Controlling the wettability of the surfaces involved turned out to be a crucial element for the control of surface adhesion and for the implementation of a completely adhesive-free production method of colloidal probes. Moreover, we present a statistical characterization protocol of the probe dimensions and roughness based on the AFM inverse imaging of colloidal probes on spiked gratings. We have assessed the influence of defects of the grating on the characterization of the probe, and discussed the accuracy of our characterization technique in comparison to the methods based on scanning electron or optical microscopy, or on the manual analysis of AFM inverse images. (C) 2011 American Institute of Physics. [doi:10.1063/1.3553499]
\end{abstract}

\section{INTRODUCTION}

Since its invention, the atomic force microscope (AFM, as for Atomic Force Microscopy) (Ref. 1) has emerged as an important tool for studying surface interactions and providing quantitative information about material properties such as elasticity, adhesion, surface potential or surface charge density, thanks to its ability to detect small forces down to the piconewton scale (for a review, see Ref. 2 and references therein). In typical AFM spectroscopic experiments (hereafter referred as force-distance experiments), the AFM probe is periodically approached to, and retracted from, the sample surface, and the tip-sample interaction force (proportional to the cantilever deflection), and/or some other interfacial properties (capacity, electric current, ... ) is measured as a function of the tip-surface distance. Generally, the most used probes in atomic force microscopy are the sharp micro fabricated silicon nitride $\left(\mathrm{Si}_{3} \mathrm{~N}_{4}\right)$ or single-crystal silicon tips. Although these probes provide a high spatial resolution in topographic mapping, there are some drawbacks in using them in quantitative force-distance experiments. First, the exact tip dimensions and geometry are typically unknown; moreover, the tipsurface complex geometry prevents from using simple analytical models of the interacting interface, such as the sphere on flat, sphere on sphere, for fitting data. Second, these tips have a very small contact area, due to their curvature radii in the range 10-100 $\mathrm{nm}$, and they can induce high stresses upon contact to the sample surface; such stresses can lead to plastic deformations, wear, and nonlinearities in the material response. Moreover, contamination and/or blunting of sharp tips can produce large relative changes in the overall

\footnotetext{
a) Author to whom correspondence should be addressed. Electronic mail: alessandro.podesta@mi.infn.it. Tel.: +390250317762. Fax: +390250317482 .
}

tip-sample interaction, making the measurements poorly reproducible and the interpretation of results not reliable.

Ducker and Butt ${ }^{3-5}$ suggested the use of new AFM probes, produced by attaching smooth micrometer-sized spheres to the AFM cantilevers. ${ }^{6,7}$ These probes are generally known as colloidal probes, and offer some advantages with respect to commercial integrated AFM tips. First, a smooth sphere on a planar surface is an ideal interfacial system, with respect to its geometry. Analytical models exist for the contact mechanics as well as for the electrostatics of this system, ${ }^{8}$ which can be used for data fitting. Second, the signal-to-noise ratio is typically higher when using colloidal probes due to the larger interaction area. Moreover, using colloidal probes it is easier to control the chemical composition of the probe simply attaching particles of different materials, and/or functionalizing the spheres both grafting chemical groups, or by suitable coatings. Eventually, in general much lower pressures are applied to the sample surface, provided tip-surface adhesion is to be kept low by working in fluid or in a controlled atmosphere. The main drawback of using colloidal probes is the reduced lateral resolution.

Different techniques have been developed for attaching microspheres to cantilevers (see Ref. 9 and references therein; see also Refs. 5, 10-12). Typically these techniques are subdivided in two classes: the immobile cantilever techniques and the cantilever-moving techniques. In the first class the most popular technique is the dual-wire technique, which requires a rather complex experimental setup (Fig. S1A in the supplementary material document ${ }^{13}$ ). A small amount of epoxy glue and a tiny droplet of a solution containing microspheres are deposited onto a glass slide. A chip with an integrated AFM cantilever is put onto the glass slide as well. Upon evaporation of the solvent, the spheres spread on the glass surface. Two thin metallic wires are attached to a micromanipulator with three degrees of freedom; the first wire is used to pick 
up a small amount of glue and release it onto the free cantilever end (Fig. S1B), then the second wire is used to pick up a single microsphere and deposit it onto the glued cantilever end (Fig. S1C). This technique requires the use of a micromanipulator external to the AFM system, the production and manipulation of thin wires typically slightly larger than the diameter of the microspheres $(10-20 \mu \mathrm{m}$ ) (some authors suggested the use of micropipettes ${ }^{11}$ ). Moreover, due to the weak capillary force due to the water meniscus forming between the wire and the microspheres it can be difficult to pick-up particles and displace them reliably. In the techniques of the second class, the cantilever is moved instead of the wires: first, its free end is dipped into a tiny patch of glue; second, a microsphere is picked up in correspondence of the glued cantilever end (Figs. S1D, S1E). These cantilevermoving techniques are faster than the dual-wire techniques because no wires production is needed; furthermore, the cantilever can be translated using the XYZ microtranslation stage implemented in most of commercial AFM. These techniques share with the dual-wire techniques the need of controlling the cleanliness of the surfaces involved, in particular, it is important to avoid that the glue spreads over the sphere surface and contaminates its apex. Furthermore, the use of adhesives for the firm attachment of microspheres to the cantilevers introduces another potential drawback: when the probe is used in liquid environment, in particular in organic solvents, a certain amount of the adhesive may dissolve and readsorb on the sample under investigation, as well as on the probe, perturbing the tip-sample interaction, and being potentially harmful for delicate biological specimens such as cells.

In order to get rid of all the issues and limitations connected to the use of adhesives, Bonaccurso ${ }^{9,14}$ developed a cantilever-moving technique for attaching a borosilicate glass particle onto a cantilever, based on the use of a mild adhesive like glycerol instead of epoxy resins. The function of glycerol is to facilitate the adhesion of the particles onto the cantilever. The probe is then heated in ambient atmosphere at about $780^{\circ} \mathrm{C}$ for $2 \mathrm{~h}$; this temperature is slightly below the softening point of borosilicate glass, which is qualitatively defined as the temperature at which a solid object begins collapsing under its own weight. Upon annealing, glycerol evaporates and the microsphere gets firmly and covalently attached to the cantilever. This technique is in principle clean and results in an epoxy-free colloidal probe. The sintering technique is applicable only to materials such as borosilicate glass, which have a melting temperature significantly lower than that of silicon nitride or silicon cantilevers $\left(1200-1900^{\circ} \mathrm{C}\right)$; moreover, care must be taken in order to avoid contamination of the microspheres, in particular impurities of the glycerol can survive after the heating step and contaminate the colloidal probe surface.

In this work we present a novel technique for the production of epoxy- and adhesive-free colloidal probes for atomic force microscopy, inspired by the work of Bonaccurso. Using a cantilever-moving approach, we exploit the capillary adhesion between borosilicate glass microspheres and AFM cantilevers to attach the sphere to the cantilever free end. A suitable modification of the adhesive properties of the glass substrate makes adhesion of spheres to the substrate weaker than that between sphere and cantilever; this in turn facilitates the detachment of the sphere from the substrate once it has been hooked by the cantilever. We then cure the cantileversphere system for a few hours at $780^{\circ} \mathrm{C}$ to bind covalently the sphere to the cantilever. The procedure is relatively quick and very reliable, and produces clean, epoxy-free colloidal probes.

We have also addressed the accurate characterization of probe dimensions and roughness. The knowledge of probe radius, in particular of the radius of the apical region of the colloidal probe interacting with the sample surface, is crucial for performing quantitative and reliable measurements with the AFM. Moreover, recent theoretical and experimental studies have shown that the roughness of the interacting region of the probe, as well as the roughness of the sample, has a strong influence on the probe/sample interaction potential. ${ }^{15-17}$ Microspheres may have very different surface roughness depending on size and material; ${ }^{18}$ accurate and reliable protocols for the evaluation of probes' roughness would be therefore a valuable support to these studies.

The characterization of colloidal probes geometry and dimensions reported in the literature is mostly based on scanning electron microscopy (SEM, as for Scanning Electron Microscope). SEM can provide accurate characterizations (relative error $\sim 1 \%$ ), but this is typically time consuming and requires using metrological equipments and procedures. Quantitative metrological measurements by SEM are not standards, this technique being not quantitative in the three dimensions, and suffering from parallax errors and distortions. ${ }^{19}$ Moreover, SEM analysis, requiring manipulation of the fragile probe and exposition to intense electron beams, can be potentially destructive for the probe and of course implies that the researchers have access to expensive SEM facilities. Other procedures to characterize the colloidal probes are based on optical microscopy, but these techniques are not very accurate (relative error $\geq 10 \%$ ) being diffractionlimited and suffering by similar parallax and optical aberration problems. The characterization of colloidal probes by AFM has been reported by Neto et al. ${ }^{20}$ This technique is based on the so-called reverse imaging of the colloidal probe on a spiked grating, which occurs when the AFM tip scans features with very high aspect ratio. ${ }^{21,22}$ The approach suggested by Neto et al. is interesting because a properly calibrated AFM can provide metrological characterization of the fully three-dimensional (3D) morphology of the interacting region of the microsphere, including the possibility of characterizing its surface roughness. The procedure described by Neto et al. is however based on the manual analysis of a few section profiles of inverted images of the probe, which impacts on the statistical strength of the analyses. Our approach in turn is based on the statistical evaluation of the geometrical parameters (height, volume, and section area) of hundreds of reverse images of the probe, and on the fitting of the experimental data using the geometrical model of the spherical cap; this protocol allows characterizing the radius of the spherical probe with precision better than $1 \%$ and accuracy determined by the calibration accuracy of the AFM, typically a few percent. In addition to the (local) radius of curvature, our protocol also allows characterizing the truly three-dimensional shape 
and the roughness of the spherical probe in the interaction region.

\section{MATERIALS AND METHODS}

\section{A. Microspheres dispersion}

Borosilicate glass microspheres with nominal radius of 2.5 and $5 \mu \mathrm{m}$ were purchased from SPI (SPI supplies, West Chester, PA 19380, USA). The microspheres are shipped as a dry powder in a small vial, therefore they must be dispersed in water. To this purpose, we first disperse an amount of powder equal to the tip of a capillary tube $(\sim 3 \mathrm{mg})$ in a droplet of alcohol; second, we add $\sim 100 \mathrm{ml}$ of HPLC-grade water and we sonicate the solution for a few minutes.

\section{B. Production of colloidal probes}

Standard rectangular glass slides for optical microscopy are first cleaned by sonication in acetone, and then coated by a $100 \mathrm{~nm}$ thick Au film using a Pabisch Top Autocoater SC-20 apparatus for sample metallization. A drop of $\sim 10 \mu$ l of the solution containing the microspheres is spotted onto the $\mathrm{Au}$ coated glass slide, and let completely dry for a few minutes. This procedure typically leads to well dispersed microspheres onto the glass slide. Using the XYZ microtranslation stage of the AFM the free end of the cantilever is brought in contact with a single sphere, which is immediately captured by the capillary adhesion force connected to the formation of a water meniscus between the cantilever and the sphere. Upon withdrawal of the cantilever, the sphere is detached from the substrate that has a lower adhesion, and the chip is transferred into the oven and kept for $2 \mathrm{~h}$ at $780^{\circ} \mathrm{C}$. After cooling of the system to room temperature, the colloidal probe is transferred back to the AFM for characterization.

\section{Atomic force microscopy characterization of substrates and probes}

For the assembling of the colloidal probes, we have used the XYZ microtranslation stage of a Nanoscope V Bioscope2 AFM (Veeco Instruments); the same instrument has been used for both the characterization of adhesion force between the colloidal probes and the glass substrate, and the characterization of the probe size and geometry by reverse imaging on a MikroMasch TGT01 spiked grating. All measurements have been performed in air (RH $\sim 40 \%)$. Scan areas for probe characterization by reverse imaging were typically 15 $\times 15 \mu \mathrm{m}^{2}$, with scan rates of $1-2 \mathrm{~Hz}$ and a sampling resolution of about $10 \mathrm{~nm} /$ pixel. Microspheres have been attached to rectangular tipless cantilevers with nominal force constants of 3 and $0.2 \mathrm{~N} / \mathrm{m}$ (NanoAndMore, $\mathrm{GmbH}$ ). For the morphological characterization of naked and Au-sputtered glass slides we have operated the microscope in Tapping Mode using single-crystal silicon tips with nominal radius of curvature of $5-10 \mathrm{~nm}$ and cantilever resonance frequency in the range 200-300 kHz. Scan areas were typically $2 \times 1 \mu \mathrm{m}^{2}$ with scan rates of $1.5-2 \mathrm{~Hz}$ and a sampling resolution of $1 \mathrm{~nm} /$ pixel.

\section{Scanning electron microscopy characterization of colloidal probes}

Scanning electron microscopy analysis of colloidal probes has been performed at the Micro and Nano-Fabrication Platform of Fondazione Filarete, Milano, Italy, using a Zeiss Sigma Field Emission Scanning Electron Microscope (FESEM). The microscope calibration has been checked previously by imaging two AFM calibration gratings with period of 10 and $1 \mu \mathrm{m}$; after calibration the FE-SEM was able to measure quantitatively the lateral dimensions of micrometric objects aligned perpendicularly to the electron beam with a relative error below $1 \%$.

\section{RESULTS AND DISCUSSION}

\section{A. Production of colloidal probes}

Three colloidal probes have been produced and characterized. In the proceeding of the article, we will refer to these probes as Probes 1-3, accordingly. In Table I, we report the relevant information about the three probes: their identification index and nominal radius, as declared by the manufacturer, as well as the radii measured by SEM and AFM, and relative discrepancies between these values (see Sec. III B).

\section{Modification of the surface adhesion of the glass substrate}

In order to reliably pick-up a microsphere with the free end of the cantilever the sphere-cantilever adhesion force must be significantly stronger than that between the sphere and the substrate. This condition is typically not fulfilled when the naked glass substrate is used. Considered that adhesion in air is due predominantly to the formation of a water meniscus between the contacting bodies, a good strategy to reduce it is to modify the effective surface energy of the interface in order to prevent the meniscus formation or to weaken its strength. Capillary adhesion force $F_{\text {cap }}$ between a sphere and a flat depends upon the contact angles $\theta_{1}$ and $\theta_{2}$ of the surfaces according to the equation ${ }^{23}$ :

$$
F_{\text {cap }}=2 \pi R \gamma\left[\cos \left(\theta_{1}\right)+\cos \left(\theta_{2}\right)\right],
$$

where $R$ is the tip radius and $\gamma$ is the surface tension of the liquid. In order to reduce sphere-substrate adhesion, we increase the effective contact angle $\theta_{2}$ of water on the glass substrate by depositing onto it a thin film of gold with thickness $100 \mathrm{~nm}$. Au coated by a tiny amount of carbonaceous contaminants has a higher contact angle with respect to glass $\left[50^{\circ}\right.$ compared to $0^{\circ}-30^{\circ}$ (Ref. 24)]. Using $\gamma_{\text {water }}$ $=0.07 \mathrm{~N} / \mathrm{m}$ and $\mathrm{R}=5000 \mathrm{~nm}$ in Eq. (1), we obtain a decrease of adhesion force on $\mathrm{Au}$ with respect to glass of only $20 \%$. The crucial point to obtain a dramatic reduction of adhesion of colloidal spheres on the substrate is the surface morphology of the sputtered film. The typical surface morphologies of the glass and $\mathrm{Au}$ surfaces are shown in Figs. 1(a) and 1(b). Glass is very smooth, with an rms roughness of only $0.6 \mathrm{~nm}$ on a $2 \times 1 \mu \mathrm{m}^{2}$ area, while sputtered gold has a roughness of $2.3 \mathrm{~nm}$, and a complex surface texture consisting in a granular network where nanometer-sized 


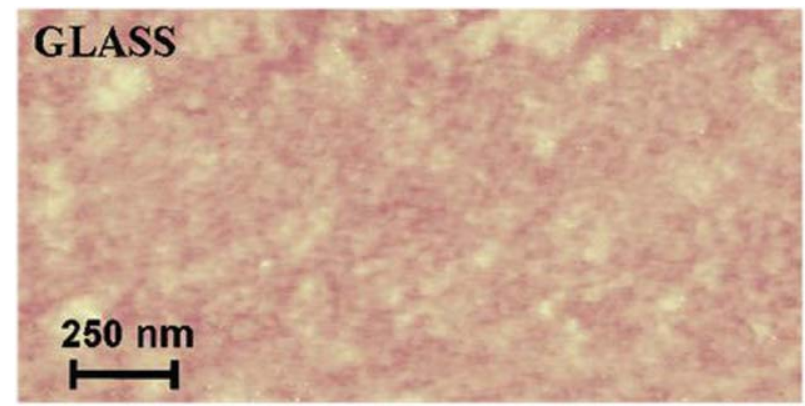

(a)

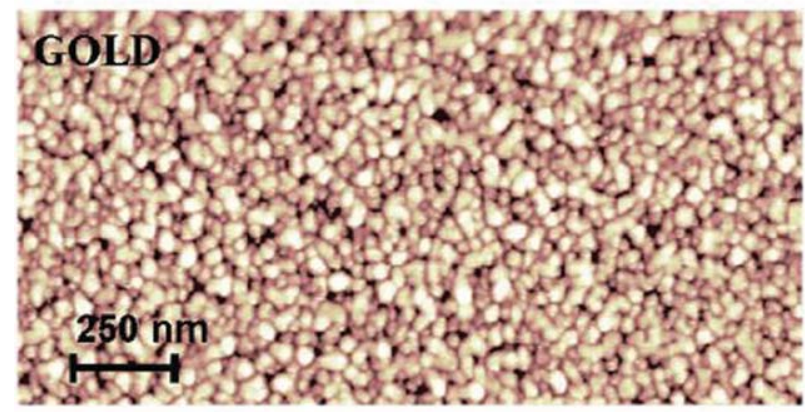

(b)

FIG. 1. (Color online) (a) AFM image of the surface of a glass slide. The rms roughness is $\sim 0.6 \mathrm{~nm}$. (b) AFM image of the surface of a thin Au film (thickness $\sim 100 \mathrm{~nm}$ ) deposited onto a glass slide by sputtering. The rms roughness is $\sim 2.3 \mathrm{~nm}$. For both images, the scan size is $2 \times 1 \mu \mathrm{m}^{2}$, the vertical color scale is $10 \mathrm{~nm}$ (height of brighter points is higher).

domains are separated by steep trenches and overhangs. Such morphology is effective in determining an increase in the contact angle of hydrophobic, but also mildly hydrophilic surfaces like gold. The underlying mechanism is the formation of air pockets at the liquid/solid interface,,$^{25}$ an effect that is enhanced on high-specific area, nanoporous surfaces. ${ }^{26,27}$ Figure S2 in the supplementary material document shows a scheme of the cantilever/sphere/substrate system, highlighting the Au-induced weakening of the capillary adhesion force. We have measured the adhesion force between the borosilicate glass microspheres and the naked and Au-coated glass surface collecting force-distance curves on the two surfaces using a freshly prepared AFM colloidal probe [Figs. 2(a) and 2(b)]; the comparison of the two representative force-distance curves shows that sputtering gold onto the glass slide reduces adhesion between the microsphere and the substrate by more than ten times. Using the same colloidal probe, we have investigated the surface adhesion at the boundary between the sputtered $\mathrm{Au}$ film and the naked glass substrate by Force-Volume imaging. ${ }^{2}$ Force-Volume consists in recording force-distance curves along a grid spanning a finite area. From each force-distance curve the local relative height of the surface can be calculated, as well as the local adhesion, corresponding to the pull-off force, i.e., the depth of the adhesion well in the force-distance curves. Topographic and adhesion maps extracted by the Force-Volume data are shown in Fig. 2(c). Comparing topography and adhesion maps we can see that the region with lower adhesion corresponds to the $\mathrm{Au}$ film. Deposition by sputtering of a thin Au film turns out
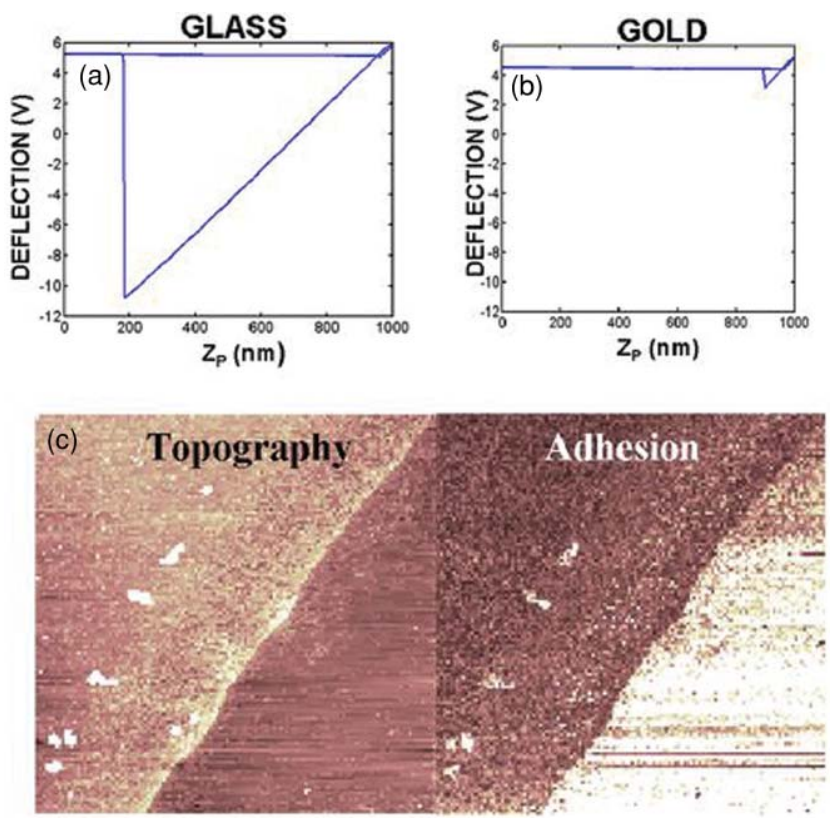

FIG. 2. (Color online) Representative force curves acquired using a colloidal probe with nominal radius $2.5 \mu \mathrm{m}$ onto (a) the glass slide and (b) the Aucoated glass slide. Adhesion between the colloidal probe and the substrate is more than ten times weaker on gold than on glass. (c) Topography and adhesion maps recorded in Force-Volume mode at the glass/Au boundary on the glass slide. Adhesion is stronger on brighter spots (corresponding to the glass surface). The scan size is $10 \times 10 \mu \mathrm{m}^{2}$, the vertical color scale is 20 $\mathrm{nm}$ (topography), and $200 \mathrm{nN}$ (adhesion map).

to be an effective strategy to reduce the adhesion between microspheres and substrate and therefore to facilitate the capture of the spheres by the cantilever free end. Different strategies can be devised in order to modify the adhesive properties of the substrate, such as chemical functionalization using some hydrophobic moieties; sputter coating has the advantage of being a rather quick and reproducible procedure, providing clean, low-adhesion substrates in less than $10 \mathrm{~min}$.

\section{B. Characterization of colloidal probes}

\section{SEM characterization of colloidal probe radius}

Figure 3(a) shows a SEM image of a borosilicate glass microsphere attached to a tipless silicon cantilever using the epoxy-free procedure that we have described in Sec. II (Probe 1). Due to parallax effects, the alignment of the cantilever-sphere assembly with respect to the electron beam is critical for the accurate assessment of the sphere radius. SEM images taken from a side like the one shown in the main frame of Fig. 3(a) are used to quantitatively evaluate the probe radius. The more reliable way to proceed is to fit an arc of circumference to the digitized sphere profile. Digitization is performed using custom MATLAB routines that convert well-contrasted, gray-scale SEM images into black and white images, where the white spherical probe neatly rises up from the black background. The pixels where the contrast reversal takes place define the profile of the sphere. For each Probes 2 and 3 different side views have been acquired by SEM, and the sphere profiles have been digitized and fitted. Figure 3(b) shows a representative 

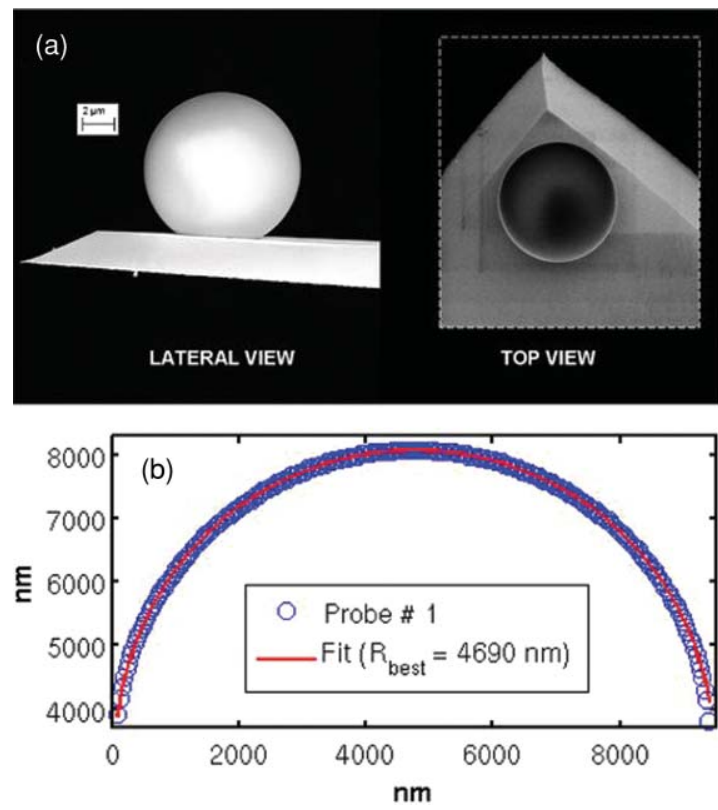

FIG. 3. (Color online) (a) SEM images (side and top views) of Probe 1 produced using the epoxy-free protocol. (b) A digitized profile extracted from a side-view SEM image, fitted by an arc of circumference.

digitized profile with the fitting curve, from which the radius of the probe is extracted. In the third column of Table I we report the average values (with standard deviations of the mean) of the radii measured by SEM of Probes 1-3. The same probes have been characterized by AFM reverse imaging.

\section{Basic principles of the statistical characterization protocol of colloidal probes by AFM reverse imaging}

When scanning the AFM tip across a surface decorated by features with very high aspect ratio, convolution of the probe geometry to the sample morphology deeply affects the imaging process. The MikroMasch TGT01 calibration grating consists of an array of sharp spikes with tip radius less then 10 $\mathrm{nm}$ and tip angles below $25^{\circ}$ [Fig. 4(b)]. The lateral and diagonal separations of the spikes are 2.12 and $3 \mu \mathrm{m}$, accordingly, and the height of the spikes is in the $600-800 \mathrm{~nm}$ range. Using the TGT01 grating, convolution is pushed to its maximum and the result is the inverted imaging of the AFM probe ${ }^{21,28,29}$; inverted images of the probe can be modeled by spherical caps. Figures 4(a) and 4(c) show the result of scanning a spherical tip with nominal radius $R=5 \mu \mathrm{m}$ across the TGT01 grating: the image consists of an array of spherical caps, representing

TABLE I. Nominal and measured radii of the three probes analyzed in this study. Comparison of AFM and SEM measurements is shown.

\begin{tabular}{lclcc}
\hline \hline Probe & $\begin{array}{c}R_{\text {NOMINAL }} \\
(\mathrm{nm})\end{array}$ & $\begin{array}{c}R_{\mathrm{SEM}} \\
(\mathrm{nm})\end{array}$ & $\begin{array}{c}R_{\mathrm{AFM}} \\
(\mathrm{nm})\end{array}$ & $\begin{array}{c}\left|R_{\mathrm{SEM}}-R_{\mathrm{AFM}}\right| / R_{\mathrm{AFM}} \\
(\%)\end{array}$ \\
\hline 1 & 5000 & $4629 \pm 35$ & $4752 \pm 13$ & 2.6 \\
2 & 5000 & $5864 \pm 120$ & $6627 \pm 27$ & 11.5 \\
3 & 2500 & $2528 \pm 13$ & $2692 \pm 8$ & 6 \\
\hline \hline
\end{tabular}

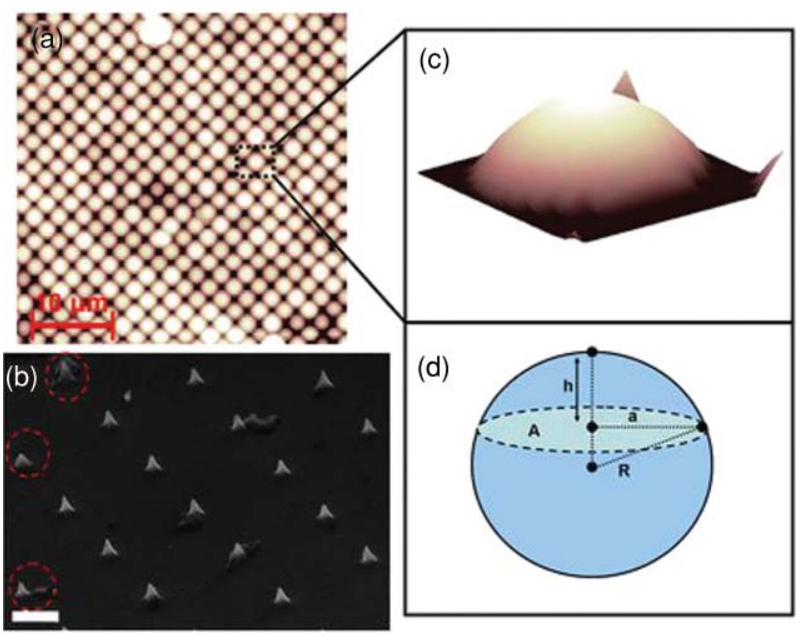

FIG. 4. (Color online) (a) AFM image of the TGT01 grating obtained with colloidal probe of nominal radius $R=5 \mu \mathrm{m}$. The scan size is $40 \times 40 \mu \mathrm{m}^{2}$, the vertical color scale is $100 \mathrm{~nm}$. (b) SEM image of the TGT01 grating (scale bar $1 \mu \mathrm{m}$ ). Some spikes are broken, some others have contaminants attached (circled features). (C) 3D magnified view of an inverted AFM image of the probe. (D) The spherical cap and its relevant geometrical parameters.

the regions of the probe that were in contact with each spike. The differences in the heights and diameters of the caps can be attributed to differences in spikes heights and to contaminations of the TGT01 surface [see the circled features in Fig. 4(b); the influence of broken spikes will be discussed in details in Sec. III B 3].

The geometrical properties of a spherical cap are schematically described in Fig. 4(d). The base radius $a$ and the height $h$ of the spherical cap, and the radius of the mother sphere $R$, are related by the following equation:

$$
R=\frac{h^{2}+a^{2}}{2 h} \text {. }
$$

The base area $A=\pi a^{2}$ is therefore

$$
A=\pi h(2 R-h),
$$

and the volume is:

$$
V=\frac{\pi}{3} h^{2}(3 R-h) \text {. }
$$

Combining Eqs. (2) and (4), the volume of the spherical cap can be written in terms of the area $A$ :

$V=\frac{2 \pi R^{3}}{3}\left(1-\sqrt{1-\frac{A}{\pi R^{2}}}-\frac{A}{2 \pi R^{2}}\right)\left(2+\sqrt{1-\frac{A}{\pi R^{2}}}\right)$,

where $h, A$, and $V$ can be measured for each object in the AFM maps. The resulting $A$ vs $h, V$ vs $h, V$ vs $A$ curves can be fitted by Eqs. (3)-(5) to extract the value of the sphere radius $R$ and its error.

The acquired images are processed using custom routines written in MATLAB environment. In particular we have developed an automated algorithm that is subdivided in two steps. In the first step we set a threshold height to the AFM image (Fig. S3A) and keep only those points lying above it; this produces an image (Fig. S3B) consisting in 
well isolated spherical caps, with different heights, that can be unambiguously identified, labeled, and individually analyzed. Noteworthy, the value of the threshold height does not influence the final result, because whatever the threshold level is, the selected objects will always be spherical caps with different heights and base radii, but same mother radius $R$. The threshold value is chosen to have the maximum number of isolated spherical caps in the image, with the highest average height. We have used the routines of the Image Processing Toolbox of MATLAB to label the objects. An example of the labeling procedure is shown in Fig. S3C: each pixel belonging to the same object has been assigned the same height — an integer value-different objects having different heights. For each object several morphological and shape parameters, such as height, base radius and area, eccentricity, and volume, have been calculated. Typically, the objects that are automatically analyzed are spherical caps (inverted tip images), and a variety of features originating from fragmented inverted tips images or from convolution between the tip geometry and contaminants or defects on the grating surface. Based on the morphological information acquired for each object, we can apply automatic filters that can discriminate spherical-like objects from nonspherical ones. For instance, a given volume-to-area ratio is expected for a spherical object, as well as a given eccentricity of the base area. A typical filter is obtained by requesting that the ratio between minor and major axes of the base section is above 0.65 , and that logarithms of volume and height are linearly correlated (practically, we fit data with a straight line and reject data that lie more than 2-3 variances away from that line). Objects that do not satisfy such relatively loose criteria are rejected. This automatic filtering aims to minimize the bias that a manual selection of the "good" objects made by the operator may introduce. In Fig. S4 a comparison between original and filtered data for Probe 2 is shown. Filters based on morphological constraints allow automatically rejecting spurious data, reducing the dispersion of the experimental data. We will discuss in Sec. III B 3 a finer filtering procedure that is able to get rid of artifacts due to nonideality of the spiked grating.

After the filtering procedure, in the second step we apply a nonlinear regression based on Eqs. (3)-(5) to the $A$ vs $h$, $V$ vs $h$, or $V$ vs $A$ curves, typically made of several hundreds of data points, and obtain the value of the probe radius $R$ and its error. The error of the parameter $R$ represents the $68 \%$ confidence interval and it is calculated according to the optimized strategy discussed by Lybanon. ${ }^{30}$ Our procedure also readily allows studying the overall geometry of the apex of the colloidal probe; each reverse image of the probe obtained on a single spike is saved individually and can be analyzed beyond the limits of the spherical cap model.

Neto et al. ${ }^{20}$ measured the radii of their probes by manually analyzing a few tens of section profiles of inverted AFM images acquired scanning a spiked reference grating. There are two main drawbacks in this procedure: first, the number of spheres that are analyzed is typically not very high (poor statistics), and second, one or even a few section profiles of the same sphere may not describe its shape and size accurately. By analyzing automatically many images and therefore collecting data from several hundreds independent inverted

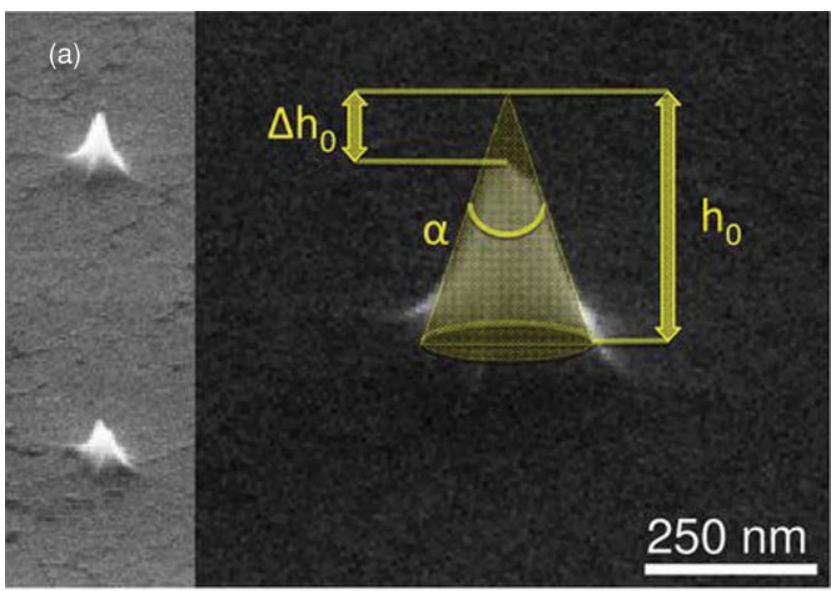

(b)

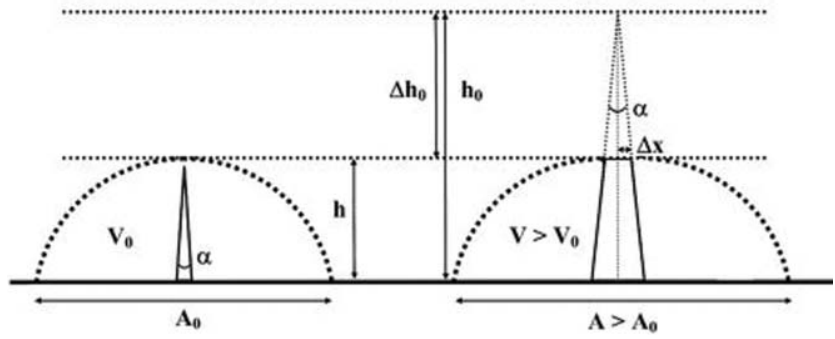

FIG. 5. (Color online) (a) SEM analysis of the spiked gratings shows that undamaged and damaged spikes coexist. An intact and a truncated spike are shown in the small box on the left. In the main frame the geometrical parameters of truncated spikes are highlighted. Average values are: $h_{0}=780 \pm$ $82 \mathrm{~nm}, \Delta h_{0}=181 \pm 55 \mathrm{~nm}$, and $\alpha=21^{\circ} \pm 3^{\circ}$. (b) A simplified model of convolution between a spherical probe and intact and truncated spikes with the same height. Broken spikes produce larger distorted spherical caps.

images of the colloidal probe, we obtain in turn statistically strong results. Moreover, we use the whole three-dimensional inverted image of the probe to calculate morphological parameters such as volume, height, and base area of each cap, therefore obtaining an accurate description of these objects.

\section{Dealing with nonideality of the spiked grating}

A closer inspection of SEM images of the spiked grating TGT01 reveals that many spikes are broken. The relevant geometrical parameters of our grating have been measured from SEM images and are (mean \pm standard deviation, $N=22$ ): $h_{0}=780 \pm 82 \mathrm{~nm}, \Delta h_{0}=181 \pm 55 \mathrm{~nm}, \alpha=21^{\circ} \pm 3^{\circ}$ [Fig. 5(a)]. The question whether this truncation may have an impact on the reverse imaging process arises. When a spherical cap-like object of height $h$ is found in an inverted AFM image, the latter could have been produced upon convolution of the probe on a perfect spike of height $h$, or on a truncated spike with the same height. In this latter case, the measured volume and area of the cap would be different from those expected for a spherical cap with the same height $h$ and motherradius $R$; fitting the $V$ vs $h$ or similar curves under these conditions would provide inaccurate estimates of the radius of the probe.

In order to understand how the imaging and data analysis process is affected by the nonideality of the grating, we 
have devised a simplified model for the reverse imaging on a defected grating [Fig. 5(b)]. Figure 5(b) shows on the left an ideal conical spike, with height $h$ and apical angle $\alpha$, and the result of AFM reverse imaging with a spherical probe: a perfect spherical cap with height $h$, base area $A_{0}$, and volume $V_{0}$ is obtained. Figure 5(b) shows on the right a broken spike with original height $h_{0}$, truncated at a distance $\Delta h_{0}$ from the apex, effective height $h$, and radius of the upper section $\Delta x$. The corresponding reverse image of the probe appears expanded with respect to the ideal one, and possesses larger volume and base area.

Using the "dilate" algorithm of the Image Processing Toolbox of MATLAB, we have simulated the convolution of a spherical probe with radius $R=1.5,2.5$, and $5 \mu \mathrm{m}$ on ideal or truncated conical spikes with $h$ in the range 40$160 \mathrm{~nm}$, and $\Delta x=0,20,40,60 \mathrm{~nm}$ [from measured $\Delta h_{0}$ and $\alpha / 2$ the average $\Delta x$ of the spikes of our grating has been calculated as $\Delta x=\Delta h_{0} \tan (\alpha / 2)=33 \pm 11 \mathrm{~nm}$; the interval 0-60 nm, according to the SEM analysis of the grating, represents approximately the $95 \%$ confidence interval of $\Delta x]$. The resolution of the images was $10 \mathrm{~nm} / \mathrm{pixel}$, comparable to that of experimental AFM images. Simulated ideal and deformed spherical caps have been analyzed using the same protocol described in Sec. III B 2, and their $V$ vs $h$ curves have been fitted in order to compare the measured radii with the nominal values. The results of the simulation are shown in Fig. 6. The values of the radii extracted by fitting the distorted data deviate from the nominal values by $7-12 \%$, the highest discrepancy being observed for the smallest probe.

The observation of the defected grating and the results of the simulation of the effects of convolution with nonideal spikes suggest that it is important to determine a practical way to assess whether the experimental data are heavily affected

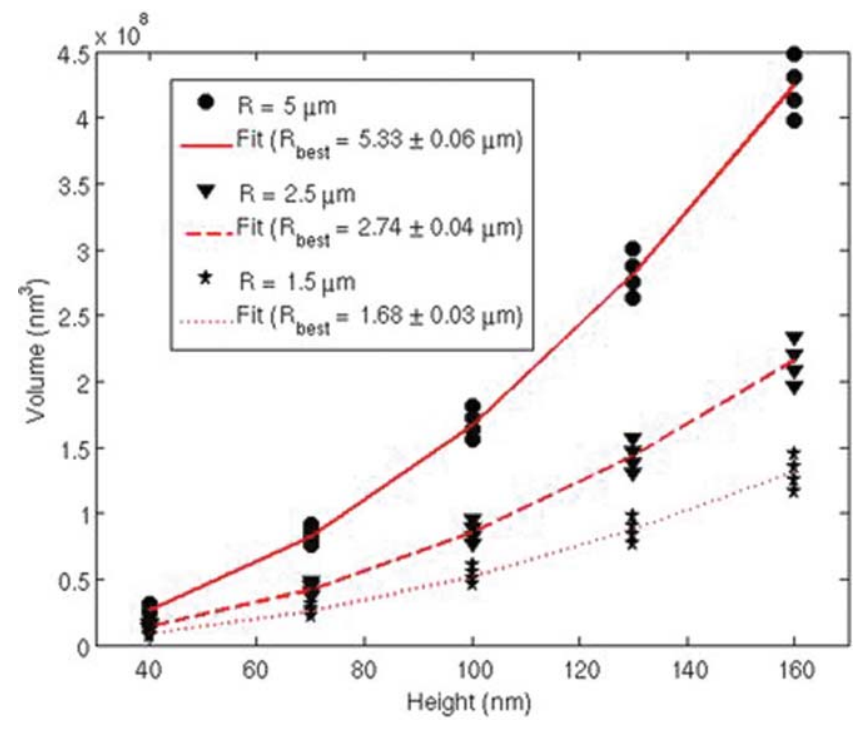

FIG. 6. (Color online) The process of reverse imaging by AFM has been simulated for probes with radii $R=1.5,2.5$, and $5 \mu \mathrm{m}$ and spikes with different heights and $\Delta x=0,20,40$, and $60 \mathrm{~nm}$, corresponding to roughly the $95 \%$ confidence interval of $\Delta x$ for the grating used in this study. With the simulated data, $V$ vs $h$ curves have been built and fitted. The relative discrepancy between nominal and fitted values of the probe radii is as large as $12 \%$. by distortions due to convolution with broken spikes, and possibly to filter off the more distorted data.

In order to have a quantitative indicator of the distortions induced by nonideality of the spiked grating we define the relative error function $\Delta V_{\text {rel }}=\left(V_{0}-V\right) / V_{0}$, where $V_{0}$ is the volume of a spherical cap with height $h$ and base area $A$ [Eqs. (3) and (4)]:

$$
V_{0}=\frac{h}{6}\left(\pi h^{2}+3 A\right)
$$

and $V$ is the measured volume. Using Eq. (6), one obtains:

$$
\Delta V_{\text {rel }}=1-6 V /\left(\pi h^{3}+3 h A\right)
$$

If the measured objects are spherical caps, as expected from the convolution of the colloidal probe on perfect spikes, $\Delta V_{\text {rel }}=0$; if in turn $h, A$, and $V$ are distorted because the spikes are broken or for some other reasons, $\Delta V_{\text {rel }} \neq 0$ represents the relative deviation of the measured volume from the ideal value. Large relative errors are a clear indication that the grating is strongly defected, or that some other issue is present; under these circumstances, we may expect that the radius extracted by the fit is not accurate. Noticeably, $\Delta V_{\text {rel }}$ can be calculated by using experimental data only, the radius $R$ not being present explicitly.

A prediction of the error introduced by convolution of the probe geometry with broken spikes can be done by comparing the spreading of $\Delta V_{\text {rel }}$ calculated for $h, A$, and $V$ values distributed normally around ideal values $h_{0}, A_{0}$, and $V_{0}$, such that $\Delta V_{\text {rel }}\left(h_{0}, A_{0}, V_{0}\right)=0$, to the spreading of $\Delta V_{\text {rel }}$ of simulated distorted caps. The variances of $h, A$, and $V$ are taken equal to the typical errors associated with the experimental data: the relative uncertainties of $V$ and $A$ are the sum in quadrature of the relative errors in $x, y, z$ (for $V$ ) and $x, y$ (for $A$ ) due to finite sampling of images $(\Delta x / x=\Delta y / y \sim 2 \%)$, and to uncertainty in the calibration of the AFM $(\mathrm{d} x / x=\mathrm{d} y / y \sim 0.25 \%, \mathrm{~d} z / z$ $\sim 2 \%$ ); the relative uncertainty of $h$ is simply $\mathrm{d} z / z \sim 2 \%$.

Figure 7 shows the comparison of $\Delta V_{\text {rel }}$ values obtained using ideal and convoluted $h, A$, and $V$ values for $R=1.5,2.5$, and $5 \mu \mathrm{m}$. The distribution of $\Delta V_{\text {rel }}$ values for noisy ideal caps ( $\Delta x=0+$ noise) is barely symmetrical around $\Delta V_{\text {rel }}$ $=0$, with a standard deviation $\sigma \approx 3.5 \%$ irrespective to the probe radius $R$. In Fig. 7 the $\Delta V_{\text {rel }}$ values of simulated caps $(\Delta x=0,20,40,60 \mathrm{~nm})$ are also shown. The signature of the distortion of caps due to convolution with broken spikes is the significant shift toward negative values of the $\Delta V_{\text {rel }}$ distribution. In the real case, the distribution of $\Delta V_{\text {rel }}$ values for distorted caps is quasicontinuous rather than discrete, because experimental errors cause a significant broadening. The stronger negative shift is observed for the smallest probe.

On the basis of the results of the simulations reported in Figs. 6 and 7, we adopt the following practical criterion to check the degree of distortion of experimental data and minimize its effect: we calculate $\Delta V_{\text {rel }}(h, A, V)$, then we reject all those objects whose $\Delta V_{\text {rel }}$ differ from zero for more than a standard deviation of the distribution calculated using nonconvoluted data $(\sigma \approx 3.5 \%)$.

Figure 8 shows the $\Delta V_{\text {rel }}$ distribution for Probes 1-3. The distribution for the Probe 1 is nearly ideal, symmetric around zero with standard deviation even smaller than $3 \%$. 

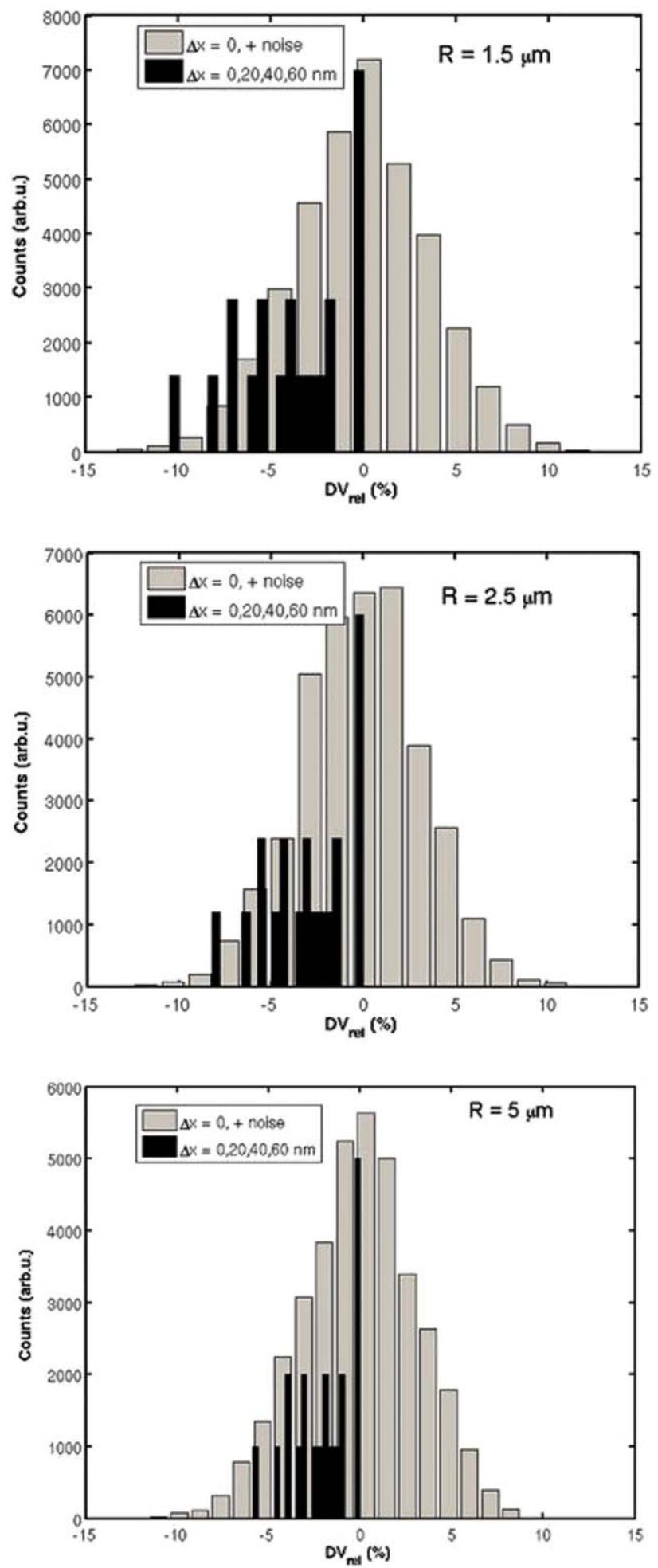

FIG. 7. The relative error function $\Delta V_{\text {rel }}(h, A, V)=1-V / V_{0}$ can be calculated for noisy ideal data (whose values are normally distributed around values $h_{0}, A_{0}$, and $V_{0}$ satisfying the condition $\Delta V_{\text {rel }}\left(h_{0}, A_{0}, V_{0}\right)=0$ ) as well as for the convoluted data shown in Fig. 6. Ideal data produce well-peaked distributions that are nearly symmetric around zero, with a standard deviation of about $3.5 \%$ irrespective to the probe radius; the signature of distortion of spherical caps is a significant shift of $\Delta V_{\text {rel }}$ toward negative values.

Only a few outliers were rejected upon applying the filtering criterion. The distribution of the Probe 2 is symmetric but shifted toward $-\infty$ by $\sim 2.3 \%$. The filtering criterion led us to reject data from the left wing of the distribution,

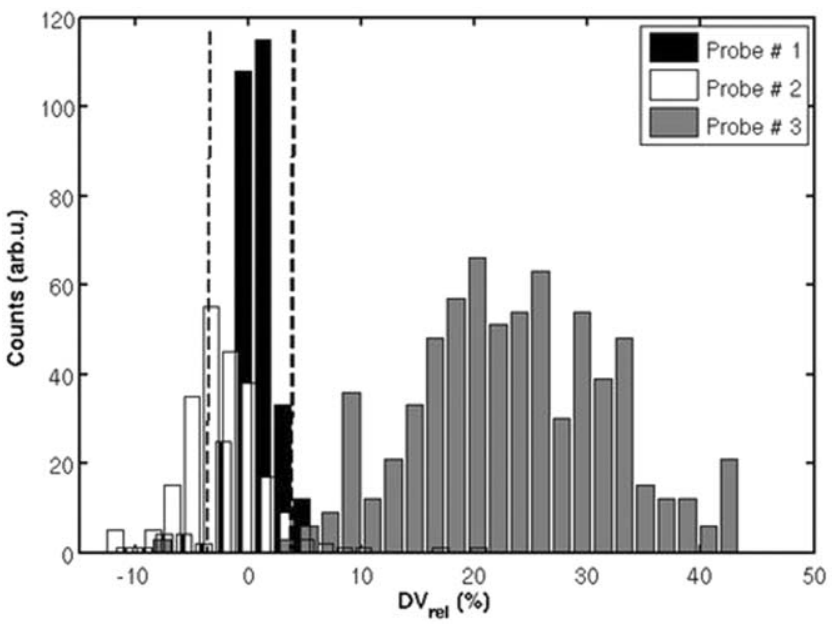

FIG. 8. The relative error function $\Delta V_{\text {rel }}(h, A, V)$ for the experimental data acquired by AFM reverse imaging of the three probes investigated in this study. While data of Probes 1 and 2 correlate well with the spherical cap model, data of Probe 3 are heavily distorted. Dotted vertical lines indicate the $\pm 3.5 \%$ interval.

corresponding to $\sim 35 \%$ of data. It may look surprising that the degree of distortion is rather low, despite the SEM analysis of the TGT01 grating revealed significantly damaged spikes. Two arguments can be invoked to explain the fact that data look better than expected. First, the grating is not uniformly damaged; on the typical scale of AFM investigations (tens of micrometers) it is possible to find either damaged or undamaged domains. Second, the geometrical model we have used for describing the convolution of a sphere with a broken spike is probably too simplified; in fact, SEM images of damaged spikes show that the apex of a broken spike is usually not flat (a circumstance that maximizes the effect of convolution, i.e., the increase of volume and base area), but instead has a quasipyramidal shape, even though the side walls are not as steep as those of an undamaged spike; convolution effects are mitigated by the presence of these secondary apical tips.

The $\Delta V_{\text {rel }}$ distribution of the Probe 3 is very broad and shifted toward $+\infty$ by more than $20 \%$. Not surprisingly, we could not fit the $V$ vs $h$ curve satisfactorily. On the contrary, the $V$ vs $A$ curve could be fitted successfully. Combining Eqs. (2) and (4) it is possible to define a new error function $\Delta V_{\text {rel }}^{\prime}(A, V, R)$ that does not depend explicitly on the height $h$ :

$$
\begin{aligned}
\Delta V_{\mathrm{rel}}^{\prime}= & 1-3 V /\left[2 \pi R^{3}\left(1-\sqrt{1-\frac{A}{\pi R^{2}}}-\frac{A}{2 \pi R^{2}}\right)\right. \\
& \left.\times\left(2+\sqrt{1-\frac{A}{\pi R^{2}}}\right)\right] .
\end{aligned}
$$

This function depends explicitly in turn on the radius $R$, which is typically not known a priori. If the value $R \approx 2.7 \mu \mathrm{m}$ is used in the calculation of $\Delta V_{\text {rel }}^{\prime}(A, V, R)$ for Probe 3 the distribution appears well peaked and symmetric around the origin [Fig. 9(a)], although with a large standard 

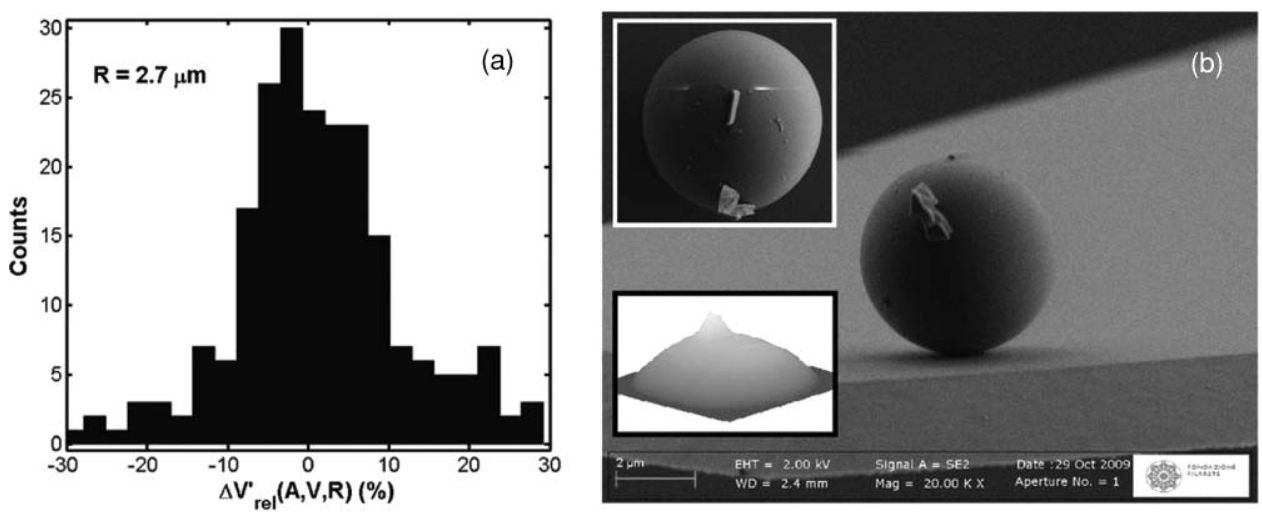

FIG. 9. (a) The alternative error function $\Delta V_{\text {rel }}^{\prime}(A, V, R)$ of Probe 3 is well peaked and symmetric around the origin, provided the value $R=2.7 \mu \mathrm{m}$ is used; this suggests that $A$ and $V$ data are correlated according to the spherical cap model, although $h$ and $V$ data are not. (b) SEM analysis of Probe 3 reveals a particle attached in the vicinity of the probe apex. The impact of the attached particle on the imaging process is shown in the bottom-left inset: a small bump is produced in the apical region of the spherical cap (detail of an AFM image).

deviation. The fact that a value of $R$ exists such that $\Delta V_{\text {rel }}^{\prime}(A$, $V, R)$ is symmetric around the origin suggests that the $A$ and $V$ values extracted by reverse AFM images of Probe 3 fit accurately to the spherical cap model, while evidently $h$ values are not reliable. The height of an object is a single-value parameter, i.e., it is calculated as the maximum height of the object, while volume $V$ and area $A$ are calculated using all the data points of each cap; it is therefore possible that well localized distortions on overall good AFM inverted images produce strongly off-trend $h$ values, while not affecting $V$ and $A$ values. Contrary to experimental best practices, Probe 3 has been used before characterization, scanning it across the surface of a soft biological specimen (human skin). The SEM analysis of Probe 3 [Fig. 9(b)] clearly shows that a particle has been picked up in the apical region. When the probe was scanned across the TGT01 grating, the attached particle behaved like a secondary small tip. Convolution of the probe geometry with the spikes of the grating produced the peculiar inverted image shown in the bottom-left inset of Fig. 9: a spherical cap with a small bump in the apical region. The spherical cap is reproduced overall satisfactorily [as witnessed by the $\Delta V_{\text {rel }}^{\prime}(A, V$, $R$ ) function], with the exception of the apical region, which is distorted, determining unexpected height values.

The analysis of the error functions $\Delta V_{\text {rel }}(h, A, V)$ and $\Delta V_{\text {rel }}^{\prime}(A, V, R)$ allowed to recognize that the experimental data of the smallest colloidal probe were partially corrupted, to identify the corrupted data $(h)$ and the good ones ( $A$ and $V$ ), to filter them [retaining only data satisfying the condition $\left(\left|\Delta V_{\mathrm{rel}}^{\prime}(A, V, R)\right|<5 \%\right]$, and to use them for fitting.

\section{Fitting AFM reverse imaging data}

Among the different curves that can be used for extracting the value of the probe radius $R$ [Eqs. (3)-(5)], we preferentially have used the $V$ vs $h$ curve, for the following reasons. First, the measurement of heights of isolated objects in the AFM is not influenced by tip/surface convolution, therefore the height data are supposed to be rather accurate. Second, the volume is calculated by using all the data of each cap, and represents a good average estimator of shape and both vertical and lateral size of the object, preferable to the base area. We have therefore fitted the $V$ vs $h$ curves of Probes 1 and 2. In the case of Probe 3, as discussed in Sec. III B 3, we have fitted the $V$ vs $A$ curve because the measured height values turned out to be not representative of the overall shape of the inverted AFM images of the probe.

Figure 10 shows filtered experimental data of the three probes and their best fit curves. In all cases the spherical cap
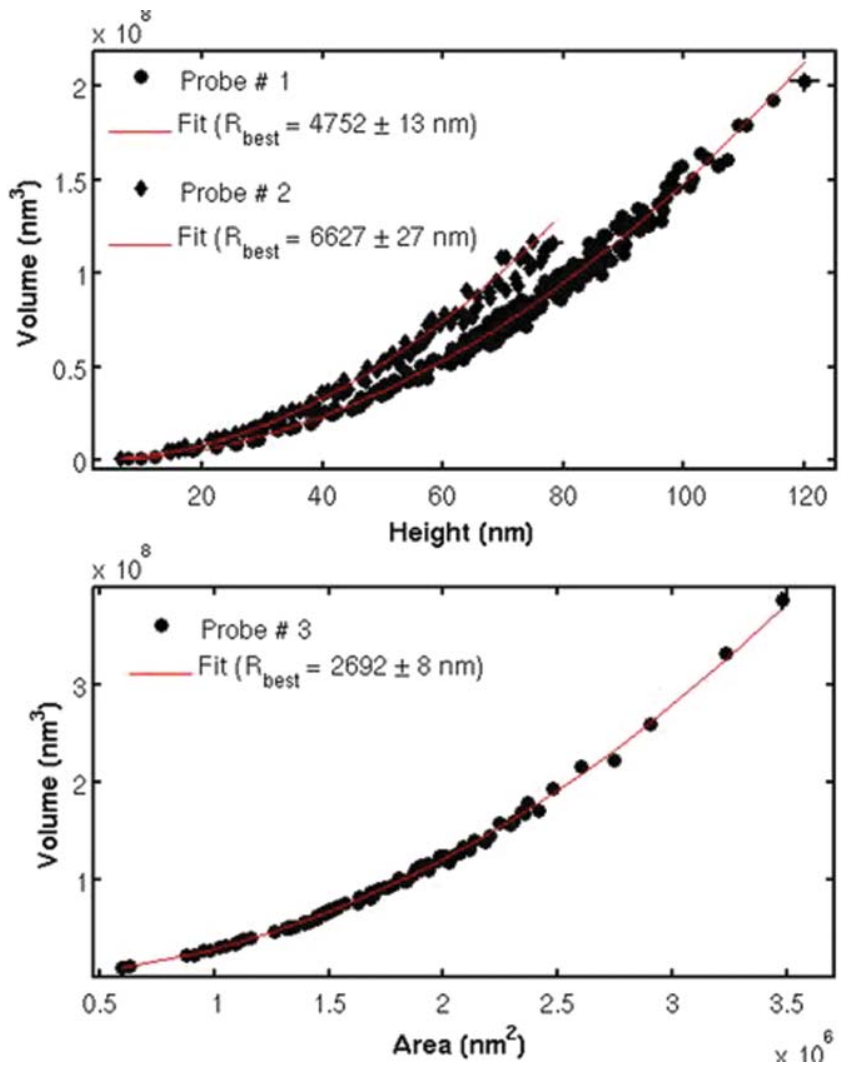

FIG. 10. (Color online) $V$ vs $h$ (for Probes 1 and 2) and $V$ vs $A$ data (for Probe 3) have been filtered first applying the basic morphological filter described in Sec. III B 2 than rejecting data with $\Delta V_{\text {rel }}(h, A, V)>3.5 \%$ [or $\Delta V_{\text {rel }}^{\prime}(A, V$, $R)>5 \%$ for Probe 3]; the agreement with the spherical cap models [Eqs. (4) and (5)] is excellent. 
models [Eqs. (4) and (5)] provided an excellent fit to the experimental data. The measured values of the radii are reported in Table I. The statistical relative errors associated with these values are very small, well below $1 \%$.

The relative discrepancy between measured and nominal values of sphere radii can be as large as $30 \%$, confirming that either the estimation of the sphere radius by the manufacturer is not accurate, or the microspheres are polydispersed to some extent, or both, and demonstrating that assuming the sphere radius being equal to the nominal radius can be an important source of error in experiments. The agreement between values of the probe radius measured by AFM and SEM is between $3 \%$ and $12 \%$, which is not very good, with the exception of Probe 1. Provided the AFM is accurately calibrated, our statistical characterization protocol is expected to provide more accurate results with respect to the SEM analysis, which is affected by alignment problems when used to image quantitatively truly three-dimensional objects; in particular it is difficult to control and check alignment of the electron beam to the sample when spherically symmetric object are imaged like AFM colloidal probes.

Noticeably, the statistical analysis protocol we have described allows getting rid of nonideality of the grating (it would be hard and time consuming to characterize it accurately, and impossible to correct-for exactly), providing an overall accuracy in the determination of the probe radius typically below $1-2 \%$.

\section{Local roughness of colloidal probes}

Each three-dimensional profile of the interacting apical region of the colloidal probe is processed in order to extract a roughness value for the probe; values of all the independent inverted images of the same probe are then averaged. Image processing consists in subtracting from each section profile of the same AFM inverted image the spherical cap best fit, then calculating the standard deviation of the subtracted profile (the profile roughness), eventually averaging all the profile roughness values. For borosilicate glass microspheres, roughness values $\leq 1 \mathrm{~nm}$ are typical, although for other materials and/or bigger sizes significantly larger values have been reported. ${ }^{18}$ Figure S5 shows a subtracted AFM inverted image of a borosilicate glass colloidal probe with nominal radius $R=5 \mu \mathrm{m}$; the residual roughness is $0.4 \mathrm{~nm}$. Probes $1-3$ have roughness of $0.26,1.1$, and $2.15 \mathrm{~nm}$, accordingly. The high roughness value of Probe 3 is due, as discussed in Sect. III B 3 [see also Fig. 9(b)], to the presence of a particle adsorbed in the apical region.

\section{CONCLUSIONS}

We have shown that epoxy- and adhesive-free AFM colloidal probes can be produced by exploiting capillary adhesion forces between borosilicate glass microspheres and AFM cantilevers, and by annealing the probes at temperatures close to the softening point of borosilicate glass. It is crucial to this purpose to control the surface wettability of both the cantilever and the substrate where the spheres are dispersed in order to make adhesion between spheres and cantilever stronger than that between spheres and substrate; this can be achieved by sputtering a few nanometers of gold onto the glass slide, thanks to the roughness-induced hydrophobic effect. The attachment of the spherical probe to the cantilever turned out to be strong and persistent; we did not observe any detachment of the probe during several adhesion, friction, and force spectroscopy experiments, in air as well as in liquid.

We have presented a novel protocol based on the statistical analysis of AFM inverse images of the colloidal probe apex obtained scanning the probes on a spiked grating, which provides accurate and reliable characterization of the radius and roughness of the probes in the contact zone; this approach is quick and reliable, and provides more accurate results than both the manual analysis of a few topographic sections of inverse AFM images, and SEM and optical microscopy. We have assessed quantitatively the effects of nonideality of the calibration grating on the determination of the probe radius. Remarkably, exploiting suitable error functions it is possible to assess the quality of the experimental data, and to get rid of distorted data, without any prior knowledge of the condition of the grating itself.

\section{ACKNOWLEDGMENTS}

This work has been supported by Fondazione Cariplo under Grant No "2007.5758-Materiali e tecnologie abilitanti 2007."

${ }^{1}$ G. Binnig, C. F. Quate, and C. Gerber, Phys. Rev. Lett. 56, 930 (1986).

${ }^{2}$ H.-J. Butt, B. Cappella, and M. Kappl, Surf. Sci. Rep. 59, 1 (2005).

${ }^{3}$ W. A. Ducker, T. J. Senden, and R. M. Pashley, Nature (London) 353, 239 (1991).

${ }^{4}$ H.-J. Butt, Biophys J. 60, 1438 (1991).

${ }^{5}$ W. A. Ducker, T. J. Senden, and R. M. Pashley, Langmuir 8, 1831 (1992).

${ }^{6}$ H. G. Pedersen, Langmuir 15, 3015 (1999).

${ }^{7}$ M. S. Hook, P. G. Hartley, and P. J. Thistlethwaite, Langmuir 15, 6220 (1999).

${ }^{8}$ H.-J. Butt, M. Jaschke, and W. Ducker, Bioelectrochem. Bioenerg. 38, 191 (1995).

${ }^{9}$ Y. Gan, Rev. Sci. Instrum. 78, 081101 (2007).

${ }^{10}$ J. Ralston, I. Larson, M. W. Rutland, A. A. Feiler, and M. Kleijn, Pure Appl. Chem. 77, 2149 (2005).

${ }^{11}$ L. H. Mak, M. Knoll, D. Weiner, A. Gorschlüter, A. Schirmeisen, and H. Fuchs, Rev. Sci. Instrum. 77, 046104 (2006).

${ }^{12}$ Attaching Particles to AFM Cantilevers, Veeco Metrology group, Support Note 226 Revision B, 2001.

${ }^{13}$ See supplementary material at http://dx.doi.org/10.1063/1.3553499. The document contains the following items: Figure S1-Experimental setup for the "dual-wire" and "cantilever-moving" techniques; Figure S2-Attaching a colloidal particle to the cantilever; Figure S3-The image analysis protocol step-by-step; Figure S4-Raw and filtered V,h data; Figure S5-Residual roughness of the interacting region of a borosilicate glass colloidal probe.

${ }^{14}$ E. Bonaccurso, Ph.D. thesis, Universität-Gesamthochschule-Siegen, Siegen, 2001.

${ }^{15}$ S. Bhattacharjee, C.-H. Ko, and M. Elimelech, Langmuir 14, 3365 (1998).

${ }^{16}$ E. M. V. Hoek and G. K. Agarwal, J. Colloid Interface Sci. 298, 50 (2006).

${ }^{17}$ R. F. Considine and C. J. Drummond, Langmuir 17, 7777 (2001).

${ }^{18}$ P. J. van Zwol, G. Palasantzas, M. van de Schootbrugge, J. Th. M. de Hosson, and V. S. J. Craig, Langmuir 24, 7528 (2008).

${ }^{19}$ J. E. Castle and P. A. Zhdan, J. Phys. D: Appl. Phys. 30, 722 (1997).

${ }^{20}$ C. Neto and V. S. J. Craig, Langmuir 17, 2097 (2001).

${ }^{21}$ L. Montelius and J. O. Tegenfeldt, Appl. Phys. Lett. 62, 2628 (1993).

${ }^{22}$ V. Bykov, A. Gologanov, and V. Shevyakov, Appl. Phys. A 66, 499 (1998).

${ }^{23}$ H.-J. Butt, and M. Kappl, Adv. Colloid Interface Sci. 146, 48 (2009).

${ }^{24}$ T. Smith, J. Colloid. Interface Sci. 75, 51 (1980). 
${ }^{25}$ J. Bico, C. Marzolin, and D. Quere, Europhys. Lett. 47, 220 (1999).

${ }^{26}$ S. Herminghaus, Europhys. Lett. 52, 165 (2000).

${ }^{27}$ A. Podestà, G. Bongiorno, P. E. Scopelliti, S. Bovio, P. Milani, C. Semprebon, and G. Mistura, J. Phys. Chem. C 113, 18264 (2009).
${ }^{28}$ K. L. Westra, A. W. Mitchell, and D. J. Thomson, J. Appl. Phys. 74, 3608 (1993).

${ }^{29}$ P. Markiewicz and M. C. Goh, Rev. Sci. Instrum. 66, 3186 (1995).

${ }^{30}$ M. Lybanon, Am. J. Phys. 52, 22 (1984). 


\title{
Adhesive-free colloidal probes for nanoscale force measurements: production and characterization
}

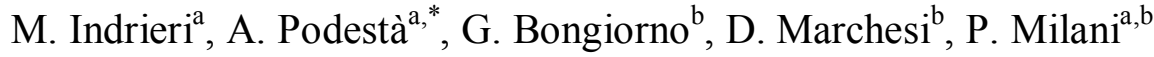

a. CIMaINa and Dipartimento di Fisica, Università degli Studi di Milano, via Celoria 16, 20133 Milano, Italy.

b. Micro and Nano-Fabrication Platform, Fondazione Filarete, Viale Ortles 22/4, 20139 Milano, Italy.

\section{SUPPLEMENTARY INFORMATION}

* Corresponding author. Tel.: +390250317762; fax: +390250317482. E-mail address: alessandro.podesta@mi.infn.it. Postal address: Dipartimento di Fisica, via Celoria 16, 20133, Milano, Italy. 


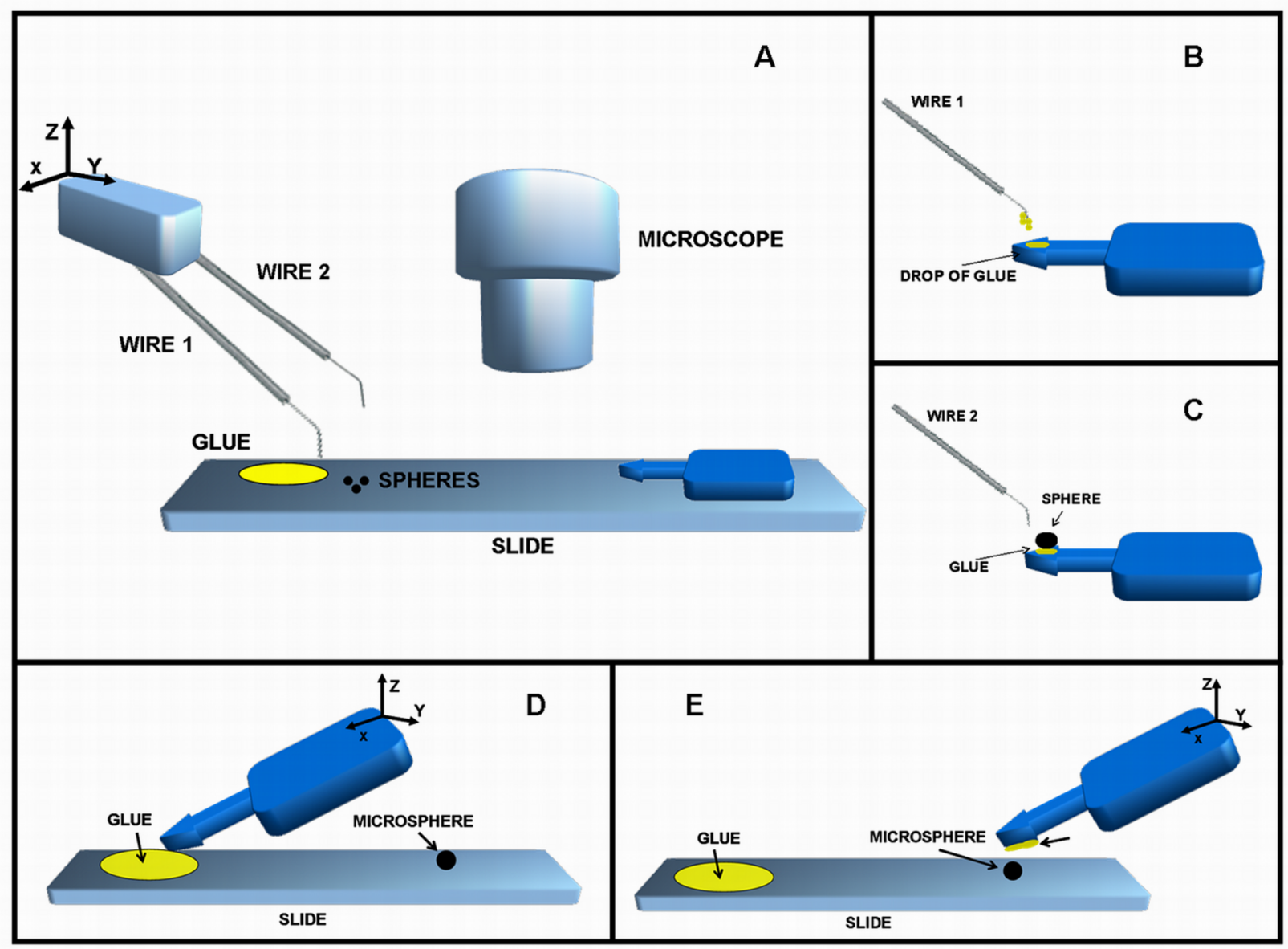

Figure S1. (A-C) Experimental setup for the dual-wire technique. The components required to attach a microsphere to a cantilever are: a micromanipulator in order to move the sharpened wires, a glass slide, and an optical microscope in order to be able to see the microspheres and the cantilever. Microspheres and a tiny droplet of glue are deposited on the glass slide; a cantilever is also positioned nearby. (B) A tiny droplet of glue is picked up by the first wire and deposited onto the cantilever. (C) One microsphere is picked up by the second wire and deposited onto the same location of the glue on the cantilever. (D-E) Experimental setup for the cantilever-moving technique. The glue and the microspheres are deposited onto the glass slide as for the dual wire technique. The cantilever is displaced across the slide and gently dipped into the glue. (E) The glued cantilever is further displaced to pick up a microsphere by its sticky end. 


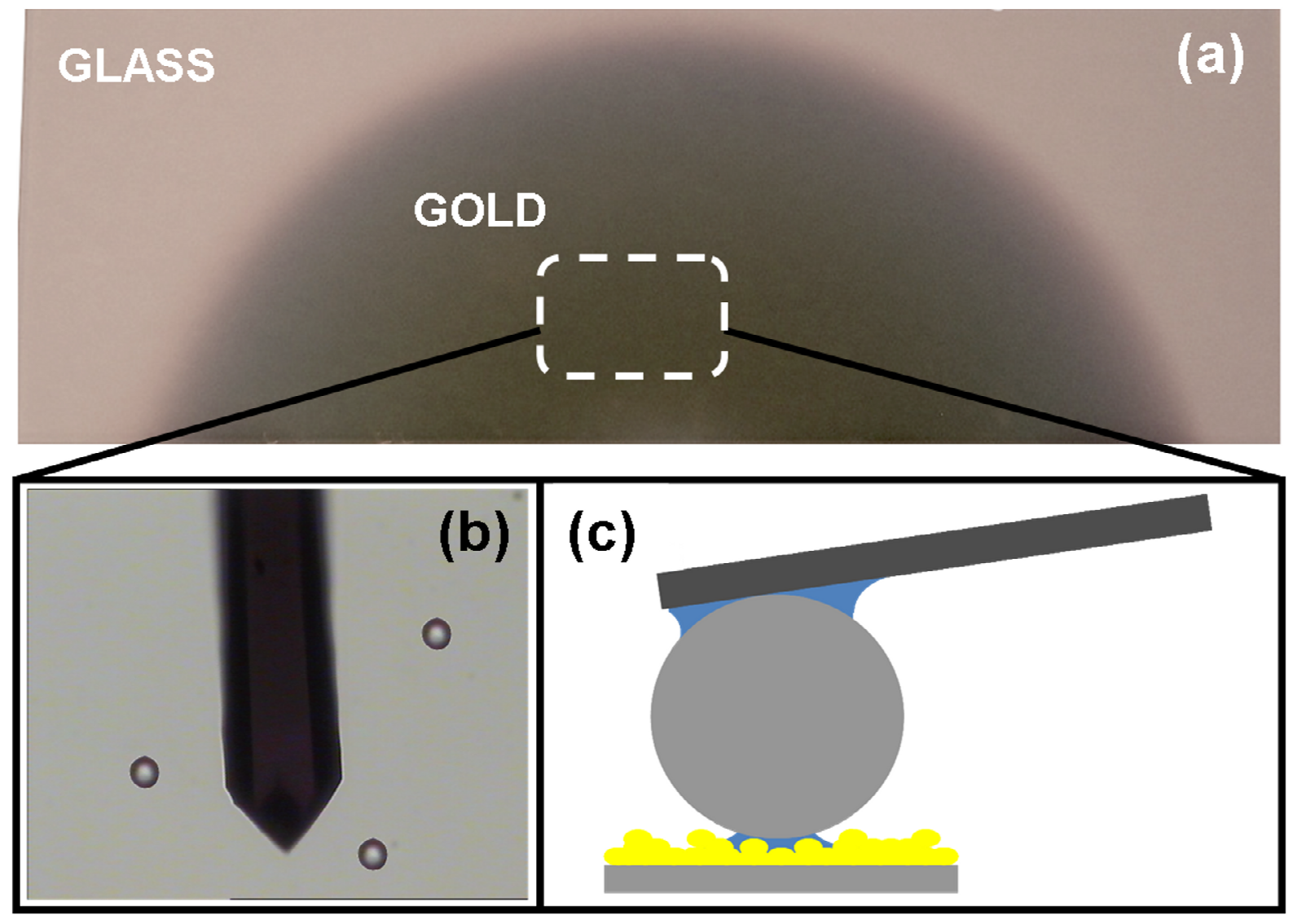

Figure S2. (a) Optical microscopy image of the Au-sputtered glass slide. Microspheres are dispersed on the slide at such a density that they can easily be approached one-by-one by the cantilever, as seen in box (b). Box (c) shows schematically the cantilever/sphere/substrate contact geometry during the picking-up process; menisci of different strength form between the cantilever and the sphere (strong adhesion), and between the sphere and the rough $\mathrm{Au}$ film (weak adhesion), favoring the detachment of the sphere from the substrate. 


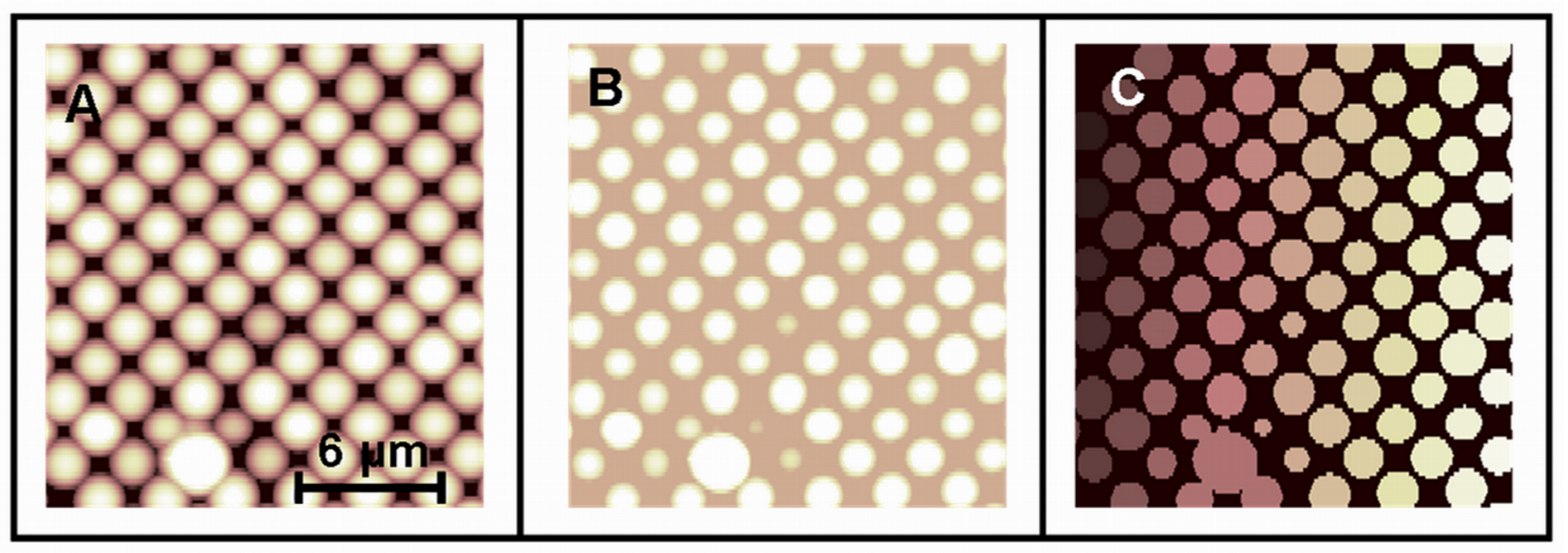

Figure S3. The image analysis protocol step-by-step. (A) AFM topography of the TGT01 grating acquired with a colloidal probe. (B) The same image after thresholding: inverted AFM images of the probe appears as spatially separated spherical caps, with different heights, base areas and volumes but (in principle) same mother radius R. (C) Each object in the image is identified and labeled; a terraced matrix is built assigning the same integer height value to the pixels of the same object, each object being assigned a different height. The resulting matrix shows a color gradient, from black (first object, upper left corner) to white (last object, lower right corner). 


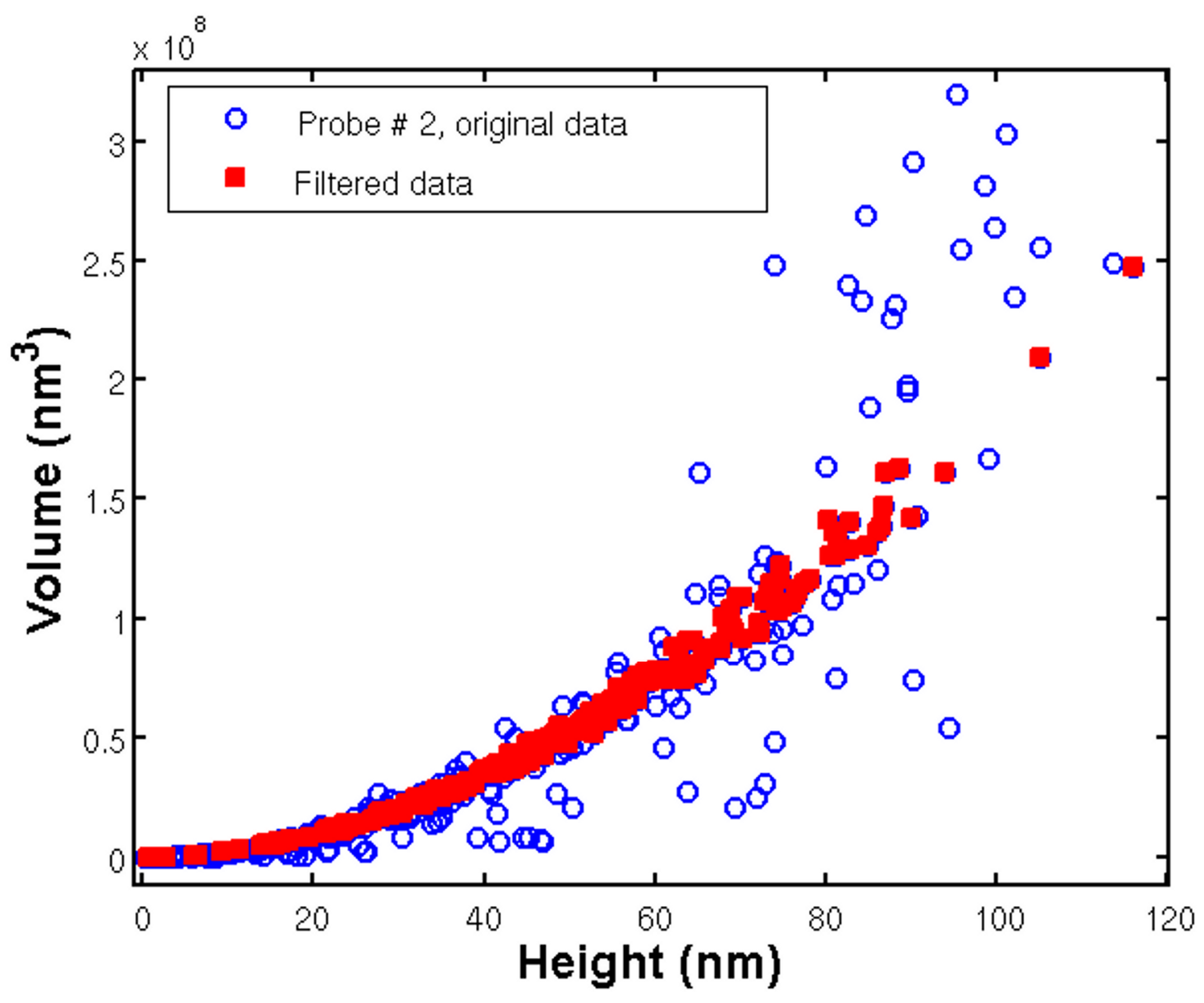

Figure S4. V,h data extracted by AFM reverse images of Probe \#2. A comparison between raw (open circles) and filtered (filled squares) data is shown. Filtering has been performed imposing relatively loose shape and size constraints to the data, as described in the main text. This filtering aims at rejecting those objects that do not possess an overall rounded shape, originating from imaging of fragments of spherical caps, contaminants, and/or imaging of defected regions of the grating. 


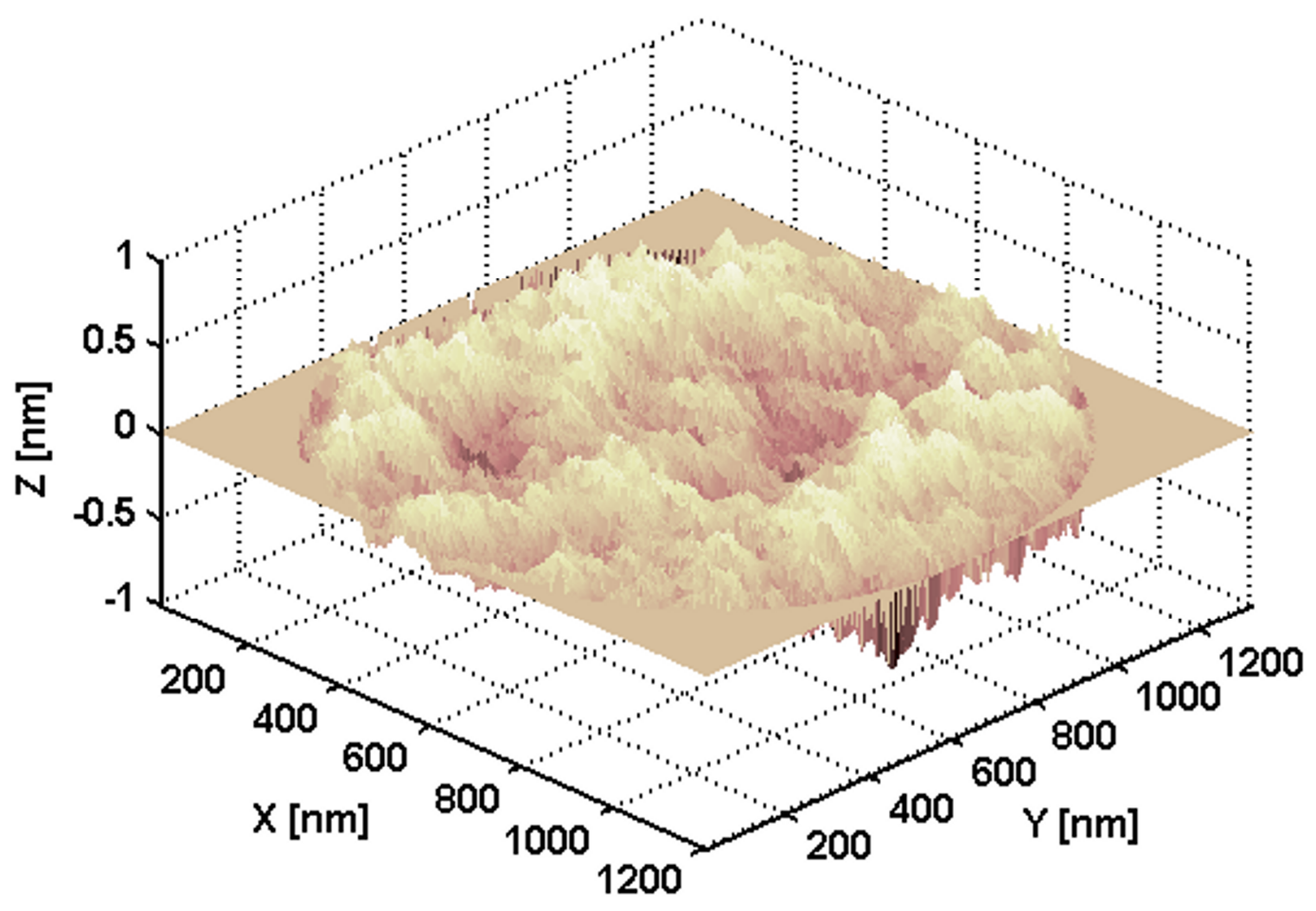

Figure S5. Residual roughness of the interacting region of a borosilicate glass colloidal probe with nominal radius of $5 \mu \mathrm{m}$. Average roughness of this probe was $0.4 \mathrm{~nm}$. This image has been obtained by subtracting one-dimensional spherical cap profiles to each profile of an original AFM inverted image of the probe. The flat part of the image represents the excluded portion (below threshold) of the inverted AFM image. Image size is $1.4 \times 1.3 \mu \mathrm{m}^{2}$, vertical scale is $2 \mathrm{~nm}$. 